\title{
EARLY STAGES OF PULSED-LASER GROWTH OF SILICON MICROCOLUMNS AND MICROCONES IN AIR AND SF 6
}

\author{
Douglas H. Lowndes, ${ }^{* 1}$ Jason D. Fowlkes, ${ }^{2}$ and Antonio J.Pedraza ${ }^{2}$ \\ ${ }^{1}$ Solid State Division, Oak Ridge National Laboratory, \\ Oak Ridge, TN, USA 37831-6056 \\ ${ }^{2}$ Department of Materials Science and Engineering, The University of Tennessee, \\ Knoxville, TN, USA 379962200
}

\begin{abstract}
Dense arrays of high-aspect-ratio silicon microcolumns and microcones are formed by cumulative nanosecond pulsed excimer laser irradiation of single-crystal silicon in oxidizing atmospheres such as air and SF,. Growth of such surface microstructures requires a redeposition model and also involves elements of self-organization. The shape of the microstructures, i.e. straight columns vs steeply sloping cones and connecting walls, is governed by the type and concentration of the oxidizing species, e.g. oxygen vs fluorine. Growth is believed to occur by a "catalyst-free" VLS (vapor-liquid-solid) mechanism that involves repetitive melting of the tips of the columns/cones and deposition there of the ablated flux of Si-containing vapor. Results are presented of a new investigation of how such different final microstructures as microcolumns or microcones joined by walls nucleate and develop. The changes in silicon surface morphology were systematically determined and compared as the number of pulsed $\mathrm{KrF}(248 \mathrm{~nm})$ laser shots was increased from 25 to several thousand in both air and SF,. The experiments in air and SF, reveal significant differences in initial surface cracking and pattern formation. Consequently, local protrusions am first produced and column or cone/wall growth is initiated by different processes and at different rates. Differences in the spatial organization of column or cone/wall growth also are apparent.
\end{abstract}

\section{Keywords}

Silicon, pulsed laser, ablation, deposition, columns, cones, whiskers, nonequilibrium growth, surface modification

* Corresponding Author:

Tel: (423) 574-6306 Fax: (423) 576-3676

E-mail: vdh@ornl.gov 


\section{Introduction: Formation of Micron-Scale Silicon Columns and Cones}

Repetitive pulsed-laser irradiation of materials at relatively low laser energy densities, $\mathrm{E}_{\mathrm{d}}$, of less than $1 \mathrm{~J} / \mathrm{cm}^{2}$ produces gradual changes in surface topography and the development of "laserinduced periodic surface structures"(LIPSS) [1,2]. Such periodic structures have been widely observed in metals, ceramics, polymers and semiconductors [3]. At the higher $\mathrm{E}_{\mathrm{d}}$ values of 1 to 5 $\mathrm{J} / \mathrm{cm}^{2}$ typically used for pulsed-laser deposition (PLD) of thin films, columnar or conical structures are formed in laser ablation targets, with the cones or columns pointing along the incident laserbeam direction $[3,4]$. Earlier studies have established connections between the laser wavelength, polarization, and incidence angle and the spatial period of the near-surface ripple structures [2], while more recent experiments have pointed out the importance of using either a short pulse duration (picosecond or femtosecond) [5] or a short (deep-UV) laser wavelength [6] for precise and efficient material removal (drilling holes or cutting trenches).

The conical structures produced by sequential laser irradiation have shapes that vary from circular cones to straight, high aspect ratio columns (see refs. 7-10 and below) as well as irregular cone clusters [3]. Most of the models used to explain the development of conical structures assume that they are formed by preferential removal of material surrounding the cones, with some sort of "cap stone" present to prevent erosion locally. The presence of impurities resistant to ablation [11], or surface modification of polymers to produce an ablation-resistant carbon layer [12], or surface segregation conducive to a transparent coating [13], all are models proposed to explain cone formation on targets irradiated in vacuum or low-pressure $(<1$ torr $)$ ambient gases.

However, we recently reported experiments in which arrays of tall, slender silicon microcolumns were formed by cumulative nanosecond pulsed-excimer laser irradiation of a silicon wafer in air [ 7 , 8]. For example, 1000 pulses of $\mathrm{KrF}(248 \mathrm{~nm})$ radiation in air produces $\sim 20-\mu \mathrm{m}$ tall Si columns with both the average column diameter and their mean separation being $\sim 2 \mu \mathrm{m}$, as shown in Figure 1. Experiments were carried out to reveal a succession of surface topographical changes as the number of laser pulses was increased and, through these, it was possible to identify the main features of the mechanism by which high aspect ratio silicon microcolumns are grown $[7,8]$. These experiments demonstrate that any explanation of microcolumn formation by cumulative pulsed excimer laser irradiation clearly requires a redeposition (not simply erosion) model, and also involves elements of self-organization within the array of columns, We note that Sanchez and coworkers also observed the formation of similar arrays of Si columns in air (see refs. 9 and 10 and the Discussion section below).

Additional experiments that we carried out recently in various ambient gases have revealed that an oxidizing atmosphere (e.g. oxygen or fluorine) must be present for columns or cones to form at all under our experimental conditions. These experiments also show that the shape of the microstructures, e.g. straight columns or steeply sloping cones connected by walls, is governed by the type and concentration of the oxidizing species (e.g. oxygen-containing air vs fluorinecontaining SF,). Based on these results it was proposed that, once a sufficient number of laser pulses have succeeded in nucleating some initial surface features (see below), then silicon columns or cones will grow rapidly by a "catalyst-free" VLS (vapor-liquid-solid) growth mechanism. This mechanism involves both the re-melting of the tips of the columns / cones and the deposition there of the intense ablated flux of Si-containing vapor that is produced with each laser pulse $[7,8]$.

The outline of this paper is as follows. We begin by briefly reviewing earlier results for the formation of fully developed but distinctly different arrays of densely packed micron-scale silicon columns or cones/walls, when cumulative pulsed-laser irradiation of silicon is carried out in air or $\mathrm{SF}$, respectively. We then present results of new experiments intended to investigate how such different final surface microstructures actually nucleate and develop. These experiments reveal significant differences in the average pulsed-laser etching rates of silicon in air and SF, as well as strikingly different processes and rates by which the first pronounced local protrusions, accompanied by local cracking or pitting of the surface, are produced. It is only after sufficiently protruding local structures have formed that the pulsed-laser driven VLS mechanism can take over and drive the subsequent growth of mature micro-column or cone arrays. In air this occurs 
quickly, but requires substantially more laser pulses in SF,. The experiments in air and SF, reveal pronounced differences in early surface cracking; in the early formation of surface patterns; in the average etching rate and redeposition of ablated materials: and in the spatial organization and simultaneity of column or cone growth. Finally, we discuss growth mechanisms and unresolved questions, and close with a brief summary.

\section{Experimental}

The experimental conditions and procedures have been described elsewhere [7]. Briefly, silicon microcolumns and cones were grown by sequential pulsed $\mathrm{KrF}$ (248 nm, $25 \mathrm{~ns}$ FWHM pulse duration) excimer laser irradiation of Si wafers, with the number of laser pulses varied from 25 to several thousand. The laser beam was focused with a lens; the irradiated area varied with $E_{d}$ but was typically $7 \mathrm{~mm} \times 2 \mathrm{~mm}$. Silicon microcolumns (Fig. 1) were found to form readily in an for $2.7 \leq E_{d} \leq 3.3 \mathrm{~J} / \mathrm{cm}^{2}$. Consequently, this $E_{d}$ was used subsequently as a standard condition while investigating the effects of other changes, e.g. different ambient gas atmospheres. The results reported below were obtained in flowing gases at $E_{d}=3( \pm 0.3) \mathrm{J} / \mathrm{cm}^{2}$ unless stated otherwise.

\section{Results}

\subsection{Pulsed-laser irradiation in air: Microcolumn morphology, crystallinity and composition}

Fig. 1 illustrates the -20 urn-long and -2-3 $\mu \mathrm{m}$ diameter silicon microcolumns that are formed in air after 1000 laser shots with $E_{d}=3 \mathrm{~J} / \mathrm{cm}^{2}$. Surface-height profiles (Figure 2a) made using a Dektak II profilometer reveal that most of the microcolumns in the center of the laser-irradiated region protrude nbove the original $\mathrm{Si}$ surface, typically by $5-15 \mu \mathrm{m}$. Scanning electron microscope (SEM) images (Figure 2b) and the surface-height profiles both reveal significant redeposition of ablated material near the edges and just outside of the laser-irradiated region. These measurements strongly suggest that a redeposition mechanism must be active during microcolumn growth. Microcolumns were formed using commercial n- and p-doped Si wafers of both (001) and (111) orientations. No dependence of microcolumn formation on the magnitude or type of doping was found [7].

Initial x-ray diffraction measurements seemed to show that the crystallographic orientation of the columns was predominantly the same as the (001)-oriented single-crystal Si substrate. However, more extensive and careful measurements [14] have revealed that (1) it is not possible to separate the (001) diffracted intensity of the columns from the much stronger background (001) intensity of an (001) silicon wafer, and (2) weak (Ill), (110) and other diffraction peaks also are present, presumably originating from the microcolumns. Hence, it appears that microcolumn growth is not epitaxial.

Energy dispersive spectroscopy (EDS) analyses performed with conventional and high resolution SEM show that the bodies of the microcolumns as well as their tips are mainly silicon. A small amount of oxygen also was detected. Auger emission spectroscopy measurements established that both $\mathrm{SiO}_{2}$ and $\mathrm{Si}$ are present very near the surface of the columns. Auger depth profiling revealed a continuous decrease of oxygen content and a pronounced increase in silicon content with sputtering time, on both the side walls and the tops of the columns $[7,8]$.

We note that Sanchez et al. have independently reported the growth of "whiskerlike" silicon column structures in air by pulsed ArF (193 nm, $23 \mathrm{~ns})$ laser irradiation of Si(100) wafers $[9,10]$. They found that the microcolumns grew in air only for a relatively the narrow range of ArF laser fluences ( $\sim 1.5$ to $\left.2.5 \mathrm{~J} / \mathrm{cm}^{2}\right)$ and that their growth was initiated only after several hundred laser pulses. They also found that the whiskedike columns grew to a height (3-30 $\mu \mathrm{m})$ that was much greater than the average depth of the laser-etched crater. Their ArF-laser etching rate in air (-1 $\mathrm{nm} /$ pulse at $-2 \mathrm{~J} / \mathrm{cm}^{2}$ [9]) is similar to the KrF-laser etching rate in our work (see section 3.3.2 below). However, their SEM observations of the silicon surface evolution prior to column 
formation differ substantially from the early stages of growth reported here, as does the microcolumn growth mechanism that they suggested (see refs. 7-10 and the discussion below).

\subsection{Pulsed-laser irradiation in SF, and other ambient gases}

The silicon surface morphology was strongly affected by the ambient gas atmosphere. A series of experiments was carried out in air, $\mathrm{N}_{2}, \mathrm{~N}_{2} / 5 \% \mathrm{O}_{2}, \mathrm{O}_{2}, \mathrm{SF}_{6}, \mathrm{Ar}$ and $\mathrm{Ar} / 4 \% \mathrm{H}_{2}$, all at atmospheric pressure. These revealed that under our standard conditions of $2.7 \leq \mathrm{E}_{\mathrm{f}} \leq 3.3 \mathrm{~J} / \mathrm{cm}^{2}$, micron-scale column or cone formation takes place only in an oxidizing atmosphere (oxygen- or fluorinecontaining in our experiments). No columns were formed in atmospheres of $\mathrm{N}_{2}, \mathrm{Ar}$ or $\mathrm{Ar} / 4 \% \mathrm{H}_{2}$ under these conditions.

Especially dramatic changes in surface morphology occurred when a silicon plasma etchant gas, $\mathrm{SF}_{6}$, was used at $1 \mathrm{~atm}$. pressure during cumulative pulsed-laser irradiation of silicon. As shown in Figure 3, long structures were produced that consisted of cones (which apparently lead the growth) joined by lower walls, which together surround deep central holes [7]. The cones formed in $\mathrm{SF}_{6}$ protrude more than $20 \mu \mathrm{m}$ above the original surface. One feature in common between the conical structures grown in SF, (Fig. 3) and the slender columns grow $\mathbf{n}$ in an oxygen-containing ambient (Fig. 1) is that both have once-molten droplet-shaped tips. However, the conical arrays formed in SF, also differ by being more complex since they show melted droplet- or thorn-shaped features not only at the tops of the cones and walls but also on their sides, as shown in Fig. 3. Another significant difference is that cone and wall structures similar to those shown in Filg 3 could be grown in $\mathrm{SF}$, at a $\mathrm{KrFE} \mathrm{E}_{\mathrm{d}}$ as low as $0.9 \mathrm{~J} / \mathrm{cm}^{2}$, which is much lower than the $-3 \mathrm{~J} / \mathrm{cm}^{2} \mathrm{E}_{\mathrm{d}}-$ value required to grow columns in air or a dilute $\mathrm{O}_{2}-\mathrm{N}_{2}$ mixture.

We note that Her et al. recently reported that similar conical "spikes" with spherical caps were produced in 500 Torr of $\mathbf{S F}_{6}$ or $\mathbf{C l}_{2}$ by irradiating silicon wafers with 500 pulses from a 100 -fs duration Ti:sapphire $(800 \mathrm{~nm})$ laser [15]. The $E_{\mathrm{d}}$-value in the $-200 \mathrm{pm}$-diameter center of their Gaussian laser beam was $-1 \mathrm{~J} / \mathrm{cm}^{2}$. However, the growth mechanism of the spherical spikes, including the spherical caps, was considered unknown in their experiments [15].

\subsection{Systematic study of the early stages of micro-column or cone formation}

Silicon microcolumns and microcones have in common the existence of apparently once-molten droplets at their tips. The fact that both oxygen and fluorine, but not inert or reducing gases, promote column or cone growth suggests that their growth process must involve chemical reactions at the transiently molten tips. However, these observations do not explain how column or cone growth is initiated, or how such different structures as microcolumns (Fig. 1) or microcones (Fig. 3) are produced after thousands of laser pulses.

In order to determine how column/cone growth is initiated, and to identify the origin of the differences due to the presence of oxygen or fluorine, the changes in silicon surface morphology were studied and compared for steadily increasing numbers of laser shots in both air and $\mathbf{S} \mathbf{F}_{6}$.

\subsubsection{Silicon surface evolution in air}

The main experimental observations in air are:

(1) After 20 to 50 pulses at $2.7 \mathrm{~J} / \mathrm{cm}^{2}$, fractures begin to develop on the irradiated surface as shown in Figure 4. For (OOl)-oriented wafers two sets of fracture lines intersect at $90^{\circ}$ to form a grid that divides the surface into rectangular blocks with sides $10 \mu \mathrm{m}$ to $40 \mu \mathrm{m}$ long. For a (111) Si surface (Fig. 4, bottom) the fracture lines definitely do not intersect at $90^{\circ}$ and occasional intersections at $60^{\circ}$ and $120^{\prime \prime}$ can be seen, as expected for three-fold symmetry. The fractures open up with increasing number of laser pulses, and deep grooves and craters develop at the fracture walls. The bases of microcolumns then start forming close to these deep grooves, which apparently trap and concentrate the laser radiation and act as emitters of ablated material. 
(2) A certain number of pulses is required to initiate microcolumn growth. For instance, as shown in Figure 5, after $400 \mathrm{KrF}$ pulses at $2.7 \mathrm{~J} / \mathrm{cm}^{2}$ only a low concentration of microcolumns has formed in air.

(3) In air the nucleation of columns is inhomogeneous, taking place always at the sides of deep grooves or pits. New columns also nucleate continuously as the number of pulses increases. Correspondingly, when irradiation is stopped after an intermediate number of pulses microcolumns with a range of heights can be observed (Fig. 1).

(4) Silicon microcolumns initially grow rapidly as the number of laser pulses increases but growth appears to halt when they reach a certain length. For example, after 600 pulses in air at 2.7 $\mathrm{J} / \mathrm{cm}^{2}$ some of the columns already have reached $-40 \mu \mathrm{m}$ height. Growth does not continue beyond this limit on additional laser pulses, but nucleation of new columns takes place continuously until a dense "forest" of Si rnicrocolumns has formed.

\subsubsection{Silicon surface evolution in SF.5}

Surface-height profiles across the center of the ablated region revealed that the silicon etching rate in $1 \mathrm{~atm}$. of $\mathrm{SF}_{6}$ was $-1.27 \mathrm{~nm} /$ puise, which is only half the etching rate of $-2.54 \mathrm{~nm} / \mathrm{pulse}$ in 1 atm. of air. For comparison, the etching rate in 1 atm. of helium was only $0.072 \mathrm{~nm} / \mathrm{pulse}$. These results clearly show the strong effect of an oxidizing ambient in increasing the average etch rate, as well as the larger effect of oxygen in comparison with $\mathrm{SF}_{6}$.

For irradiation in $\mathrm{SF}_{6}$ a dramatic difference was found in both the rate at which surface structure developed and in the nature of the surface structures themselves, in comparison with irradiation in air. Figure 6 compares the (001)Si surface evolution in $\mathrm{SF}_{6}$ and in air. The right side of Fig. 6 , for irradiation in air, shows the rapid progression from surface cracking (250 shots) to microcolumn formation near the cracks (500 shots) and rapid microcolumn growth (600 shots) that was described above. In contrast, the left side of Fig. 6 shows that for irradiation in $\mathrm{SF}_{6}$ the (001)Si surface remained fairly smooth after 250 shots, simply being uniformly etched away, except for the development of a few isolated concentric ring structures $-30-50 \mu \mathrm{m}$ in diameter.

After 500 shots in $\mathrm{SF}_{6}$, the uniform etching continued but a periodic wave pattern (wavelength -18-19 pm) appeared over the entire laser-irradiated region. Two other morphological features also appeared after 500 shots, as shown in Fig. 6 (left side). First, a nearly concentric but slightly disordered set of semi-circular ring segments formed at a number of locations, covering areas of $50-100 \mu \mathrm{m}$ for each such pattern. Second, at the points where several of these ring segments intersect, a small "dome" was found to protrude and began to grow upward with successive laser shots. The series of views in Figure 7 shows the shape of one of these disordered ring-segment patterns and the location of the dome within it.

Further laser irradiation revealed that these small domelike features actually mark the beginning of microcone growth. Additional laser shots result in the formation of deep pits on either side of the dome and ring segments, the formation of long ridges running parallel to the ring sements, and upward growth of the dome to form a microcone, which apparently then grows by redeposition of material that is ablated out of the adjacent (rapidly deepening) etch pits. In $\mathbf{S F}_{6}$ the bottom of the laser-etched region is still fairly smooth after 500 shots, with an average etch depth of $600 \mathrm{~nm} \pm 30$ $\mathrm{nm}$. However, after 750 laser shots the center of the laser-etched region is $\sim 1 \mu \mathrm{m}$ deep but with large surface-height variations of $\pm 300 \mathrm{~nm}$ or more, corresponding to the onset of pitting and cone and wall growth. We note that for $\mathrm{KrF}$ irradiation of $\mathrm{Si}$ in $\mathrm{SF}_{6}$ at $0.9 \mathrm{~J} / \mathrm{cm}^{2}$, very deep pits also are formed before conical structures appear on the originally flat $\mathrm{Si}$ surface.

\subsubsection{Effect of oxygen concentration on microcolumns size and shape}

It was discovered that the oxygen concentration of the atmosphere has a large influence on the microcolumn morphology. Smooth, straight columns were obtained when $\mathrm{KrF}$ laser irradiation was performed in air or in a $\mathrm{N}_{2}-5 \% \mathrm{O}_{2}$ gaseous mixture, whereas in pure oxygen the columns were "plastered" with globules as shown in Figure 8a, as if small droplets had been deposited onto 
the columns' vertical surfaces as well as on their tips [7]. These deposits sometimes form "canopies" that join and cover adjacent columns.

A systematic investigation of the role of oxygen in the earliest stages of microcolumn growth was carried out by measuring surface-height profiles at successive intervals of 25 laser pulses. The height profiles revealed that for irradiation in pure oxygen the silicon surface initially swells upward by $20-30 \mathrm{~nm}$ after 25, 50 and 75 laser shots, as shown in Fig. 8b. An etched central depression begins to form only after 100 laser shots, as shown in Fig. 8c.

In another experiment, pulsed-laser irradiation was done initially for 250 shots in pure oxygen, followed by 1,950 shots in air. The resulting sample displayed a mixture of the poorly formed globular structures that grow in pure oxygen (similar to Fig. 8a) together with the thin microcolumns that grow in air. The ability to control the diameter of rnicrocolumns also was demonstrated by irradiating silicon with 400 laser pulses in air, followed by 600 pulses in a 95\% nitrogen / $5 \%$ oxygen mixture. The resulting surface was covered with a mixture of the 2-3 urn diameter microcolumns grown in air plus a set of much thinner (1-2 $\mu \mathrm{m}$ diameter) microcolumns that grew in the 5\% oxygen atmosphere. These experiments show that it is possible to chemically control the diameter of microcolumns. Perhaps it will be possible to grow "forests" of columns with sub-micron dimensions.

\section{Discussion}

\section{Pulsed-laser melting of the tips of micro-columns and cones}

Model calculations show that a $\mathrm{KrF}$ laser $\mathrm{E}_{\mathrm{d}}-3 \mathrm{~J} / \mathrm{cm}^{2}$ melts a flat Si surface to a depth $-1 \mu \mathrm{m}$ [16]. The difference between the $-2 \mu \mathrm{m}$ droplet size that is observed (Fig. 1) and the calculated melt depth may be due to a lower reflectivity, and correspondingly greater absorption, of the $\mathrm{KrF}$ radiation by the Si microcolumns. The model calculations assumed a reflectivity value appropriate for a flat, virgin Si surface while the experimental reflectivity is expected to be considerably lower due to both cumulative surface roughening and to a thin surface oxide layer that acts as a partial anti-reflection coating [17].

\section{Microcolumn growth mechanism in air}

The growth mechanism for silicon microcolumns clearly is different from one involving erosionresistant column tips because the columns grow above the original surface. The cumulative thermal expansion model described by Kelly and Rothenberg [18] is not applicable because the microcolumn formation and growth observed here is strongly dependent on the ambient atmosphere. In addition, their model would not yield any significant column growth for silicon because thermal expansion in the liquid is mostly compensated by the decreased volume (increased density) of liquid silicon relative to solid silicon [19].

Based on the results presented here and previously $[7,8]$, we postulate that the Si microcolumns, once formed, grow as material is deposited on their molten tips. Earlier studies of silicon whisker growth by the VLS method [20,21] also shed some light on the mechanism of pulsed excimer laser growth of silicon rnicrocolurnns and conical structures. In conventional VLS growth, Au clusters are distributed on a Si surface that is heated to a temperature significantly above the Au-Si eutectic at $363^{\circ} \mathrm{C}$. The Au clusters alloy with the silicon forming a compound that has a melting point lower than the ambient temperature. When a silicon-rich vapor $\left(\mathrm{SiCl}_{4}, \mathrm{SiH}_{4}\right)$ is passed over the molten islands, Si dissolves into the melt at its upper surface and precipitates out of solution at the bottom. The result is that a Si whisker grows with the (molten) Au-Si compound remaining at its tip.

In the laser-driven microcolumn growth described here, each laser pulse remelts the tips of the columns. Tbe sides of the columns do not melt owing to the low laser $E_{d}$ incident upon them. Each laser pulse also produces melting and an intense flux of silicon-rich vapor from the surface 
regions between microcolumns. As in conventional VLS growth, the molten tip on top of a solid column acts as a preferred site for silicon deposition. The kinetics of deposition at the droplets is strongly accelerated because the liquid has a high accommodation coefficient [22] and efficiently catalyzes the reaction [21]. If we assume that a silicon droplet remains molten for $200 \mathrm{~ns}$ [16] and that a microcolumn grows $20 \mu \mathrm{m}$ in 200 laser pulses, then the microcolumn growth rate is $100 \mathrm{~nm}$ $1200 \mathrm{~ns}$ or $0.5 \mathrm{~m} / \mathrm{s}$. This high growth rate is the same order of magnitude as the crystal regrowth velocity during pulsed laser annealing of ion implanted silicon [16]. The silicon source for microcolumn growth is the laser-ablated silicon-rich vapor.

\section{Microcone growth in $S F_{6}$}

The major difference between air and $\mathrm{SF}_{6}$ in these experiments is the much gentler and slower surface evolution that results from pulsed-laser irradiation in $\mathbf{S F}_{6}$. As shown in Fig. 6, there is no surface cracking in $\mathbf{S F}_{6}$, but only the gradual development (with increasing number of laser shots) of a surface-wave pattern that is increasingly interrupted by intersecting semi-circular ring segment patterns $\sim 50-100 \mu \mathrm{m}$ in diameter. At points where several ring segments intersect, a small dome is formed and grows upward with successive laser shots, i.e. microcone formation begins. As noted above, additional laser shots eventually produce deep pits on either side of the dome and ring segments, and the long walls that join neighboring microcones apparently grow up from the original ring segments. The pits concentrate the incident laser flux and they also serve as intense sources of ablated material, just as do the cracks formed in air.

Perhaps the most striking difference between microcolumn growth in air and microcone growth in $\mathrm{SF}_{6}$ is that the microcolurnns can nucleate and grow at nearly any stage of laser-irradiation in air. In contrast, in $\mathbf{S F}_{6}$ it is necessary for a surface-ripple pattern to develop more-or-less uniformly over the entire surface, accompanied by a high areal density of intersecting semi-circular ring segments. It is only after this quasi-periodic pattern is formed that microcones, surrounded by deep pits and joined by lower walls, are formed. Thus, there is a strikingly cooperative aspect to microcone growth in $\mathbf{S F}_{6}$ that is absent in air, apparently because cracks form so readily in the silicon surface when it is irradiated in air. The surface-height profiles in pure oxygen as well as the Auger depth-profiling of oxygen strongly suggest that the surface cracking in air is due to silicon oxide formation in the near-surface region.

Just as for the microcolumns formed in air, pulsed-laser melting of the microcone tips produces frozen-droplet features. The additional molten-droplet features formed on the sides of the cones in $\mathrm{SF}_{6}$ could be due to redeposition there of ablated liquid silicon drops, or to repeated remelting and growth of initially small features that protrude out from the sloping cone side walls. A highresolution SEM image (Figure 9a) shows groups of small, bright features just beginning to form on the side walls of the etch pits after 500 laser shots in $\mathrm{SF}_{6}$, i.e. just when the central dome also begins to grow. It appears that such groups of protruding features, on what will become the cone sidewall, actually evolve into the many small "lateral cones" or "thorns" seen on the sides of the fully developed microcones in Figs. 3 and $9 \mathrm{~b}$. Thus, the small lateral cones apparently form at about the same stage as the center of the cone. Once formed, these lateral features are exposed to the incident laser radiation and so also can be re-melted and grow from the ablated flux on successive laser shots.

The idea that oxygen behaves in these experiments as a much stronger etchant of silicon than $\mathrm{SF}_{6}$ is supported by an experiment in which a silicon wafer was irradiated with 2000 laser pulses in an oxygen pressure of only 0.76 torr $\left(\sim 10^{-3} \mathrm{~atm}\right.$.). As shown in Figure 10, this produced a surface topography that is closer to that seen at an early stage of silicon-surface evolution in pure $\mathrm{SF}_{6}$ (see Fig. 6 for comparison). In fact, the image in Fig. 10 also is similar to the surface pattern produced by Sanchez et al. [10] by ArF-laser irradiation of silicon in 1 atrn. of air for an intermediate number of laser pulses.

Sanchez et al. ascribed the formation of silicon microcolumns in air (by $-2 \mathrm{~J} / \mathrm{cm}^{2}$ ArF-laser irradiation) to a "hydrodynamical process" that is not described in more detail $[9,10]$. They also suggested that silicon's expansion during solidification is responsible for the gradual buildup of an 
elevated reticular network that has a mesh size of microns, early in the silicon surface's evolution [10]. After a sufficient number of laser pulses this network breaks up to form isolated hillocks, from which microcolumns can grow [IO]. The early evolution of the silicon surface seen in air in our work is quite different. Instead of a reticular network we observe cracking of the silicon nearsurface region and the relatively rapid formation, adjacent to the cracks, of protrusions that become the bases from which microcolumns grow by the laser-assisted VLS mechanism that we have proposed. We note that this mechanism should apply equally well to account for the micmcolumn growth seen in air by Sanchez et al., once the initial protrusions (or "hillocks") have formed. We also note that the expansion of silicon upon solidification could also play some role during the micmcolumn growth stage by continually thrusting the center (last-to-solidify part) of the microcolumn upward with each successive melting and solidification event.

The roles of oxygen and $\mathrm{SF}_{6}$ during the microcolumn growth stage can be understood as silicon etchants that produce silicon-containing molecules that can easily attach to the molten droplet at the tip of a microcolumn (or cone). Both the liquid droplet at the tip of a column and the clusters and/or molecules that feed growth at the column tip are produced essentially simultaneously by pulsed-laser irradiation, resulting in a new type of catalyst-free VLS growth.

In closing, we note that arrays of high-aspect-ratio silicon microcolumns may be useful as lighttrapping anti-reflection coatings or as tips for geometrically enhanced (cold) field emission of electrons. For field emission the tips would need to be sharpened by plasma etching or by oxidation/wet etching in order to produce the nanometer-scale features necessary to increase the geometrical field-enhancement factor and reduce the emission threshold field.

\section{Summary and Conclusions}

Dense arrays of silicon micmcolumns or microcones are formed by cumulative nanosecond pulsed excimer laser irradiation of silicon in air or other oxidizing atmospheres. Both microcolumns (formed in air) and microcones joined by lower walls (formed in SF, ) extend well above the initial silicon surface. Their morphology and growth are strongly affected by the ambient gas environment. The experiments described here provide considerable insight into the early surface evolution as well as the nucleation and subsequent growth mechanisms of such different microstructures under our standard experimental condition of pulsed-KrF irradiation at $3 \mathrm{~J} / \mathrm{cm}^{2}$.

Imadiatiomerarace cracking occurs within 20 to 50 laser pulses and grooves open up with increasing laser irradiation. The bases of what will become microcolumns are deposited close to the grooves, which serve to focus the laser radiation and produce a locally intense ablated flux of Si-rich vapor. Auger profiling and surface-height pmfilometry suggest that the cracking may result from silicon oxide formation. In air the nucleation of microcolumns is inhomogeneous; new columns nucleate continuously as the number of laser pulses increases. Once nucleated, the silicon microcolumns initially grow rapidly but growth halts when they reach a height $-40 \mu \mathrm{m}$. The oxygen concentration has a strong effect. In pure oxygen the silicon surface initially swells upward. Laser irradiation of silicon in pure oxygen produces globular structures, while fine, straight microcolumns (-2-3 $\mu \mathrm{m}$ in diameter) are obtained in air (-20\% oxygen), and a $5 \%$ oxygen/95\% nitrogen mixture yields even smaller diameter microcolumns than in air. Thus, it may be possible to chemically control the diameters of microcolumns.

Irradiation in SF. In $\mathbf{S F}_{6}$, both the rate of development and the type of surface microstructure are quite different than in air. There is no evidence of initial surface cracking. Instead, a periodic wave pattern is formed, accompanied by an increasing density of intersecting semi-circular ring segments. At the points where several ring segments intersect a small dome forms and then grows upward on successive laser pulses, marking the beginning of micmcone growth. Additional laser shots produce deep pits to the sides, and the long walls that join microcones appear to grow up from the intersecting ring segments. A major difference between air and SF, is the gentler and slower surface evolution in the latter. In $\mathrm{SF}_{6}$ there is a cooperative aspect of surface evolution that is much less apparent in air under our experimental conditions. 
Growth mechanism. The growth mechanism for micro-columns and for cones joined by walls must involve redeposition (not merely erosion) since they grow well above the surrounding surface. It is proposed that growth occurs through a combination of pulsed-laser melting of the tips of the columns/cones/walls and redeposition there of the intense flux of Si-rich vapor produced by ablation of the surface region between columns (especially from pits or grooves). The molten tips of the columns are strongly preferred sites for deposition because the transient liquid efficiently catalyzes the growth reaction, resulting in an estimated tip growth rate $-0.5 \mathrm{~m} / \mathrm{s}$. The role of oxygen or SF, during the growth stage is to produce silicon-containing molecules that can easily attach to the transiently molten droplets at the tips of columns/cones/walls.

The overall process is conceptually similar to the VLS (vapor-liquid-solid) method for silicon whisker growth except that it is catalyst-free: no impurity is needed to form a eutectic. Instead, the pulsed laser radiation plays two roles simultaneously, both melting the tips of the columns and providing the flux of silicon-containing molecules.

\section{Acknowledgments}

The authors thank P. H. Fleming for assistance with sample preparation. We would also like to thank Professor J. L. Morenza for bringing his group's work to our attention. This research was sponsored by the Oak Ridge National Laboratory, managed by Lockheed Martin Energy Research Corp., for the U.S. Department of Energy, under contract DE-AC05-960R22464. 
Birnbaum, J. Appl. Phys. 36, 3688 (1965).

2 the series of three papers by J. F. Young, J. E. Sipe, J. S. Preston, and H. M. van iel, Phys. Rev. B 27, 1141 (1983); Phys. Rev. B 27, 1155 (1983); Phys. Rev. B 30, 01 (1984).

e S. R. Foltyn, p. 89-113 in Pulsed Laser Deposition of Thin Films (D. B. Chrisey and K. Hubler, eds.) Wiley-Interscience, New York, 1994.

R. Foltyn, R. C. Dye, K. C. Ott, E. Peterson, K. M. Hubbard, W. Hutchinson, R. E. uenchausen, R. C. Estler, and X. D. Wu, Appl. Phys. Lett. 59, 594 (1991).

Koster and K. Mann, Appl. Surf. Sci. 109-110, 428 (1997).

R. Herman, K. Beckley, B. Jackson and K. Kurosawa, p. 86-97 in Excimer Lasers. tics, and Applications, Proc. SPIE 2992, SPIE-The Int. Soc. for Opt. Eng., 1997.

J. Pedraza, J. D. Fowlkes, and D. H. Lowndes, Appl. Phys. Lett. 74, 2322 (1999).

H. Lowndes, V. I. Merkulov, A. J. Pedraza, J. D. Fowlkes, A. A. Puretzky, D. B. zohegan, and G. E. Jellison, Jr., Symposium On Surface Engineering: Science and chnology, Proc. of TM S Annual Meeting (San Diego, CA, Feb. 28-Mar. 4, 1999), The inerals, Metals and Materials Society, Warrendale, PA, in press.

Sanchez, J. L. Morenza, K. Aguiar, J. C. Delgado, and M. Varela, Appl. Phys. A 66, 83 998).

Sanchez, J. L. Morenza, R. Aguiar, J. C. Delgado, and M. Varela, Appl. Phys. Left. 69, 20(1996).

.E. Dyer, F.D. Jenkins, and J. Sidhu, Appl. Phys. Lett. 49, 453 (1986).

J. Kragnovich and J.A. Vazquez, J. Appl.Phys. 73.3001 (1992).

.I. Givargizov, J. Crystal G rowth 20, 217 (1973).

-H. Her et al., Appi. Phys. Lett.. 73, 1673 (1998).

F. Wood and G.E. Jellison, Jr., p. 165 in Semiconductors and Semimetals Vol. 23 (R.F. food, C.W. White and R.T. Young, eds.) Academic Press, Orlando, FL, 1984.

he index of refraction of $\mathrm{SiO}_{2}$ at $248 \mathrm{~nm}$ is $\mathrm{n}(248)=1.508$ so the $\mathrm{SiO}_{2}$ thickness required to rm a maximally $\mathrm{KrF}$-absorbing coating on silicon is only $-41 \mathrm{~nm}$.

. Kelly and J.E. Rothenberg, Nucl. Instr. and M ethods in Phys. Res. B 7/8, 755 (1985). Kelly and A. Miotello, p. 55-87 in Pulsed Laser Deposition of Thin Films (D. B. Chrisey nd G. K. Hubler, eds.) Wiley-Interscience, New York, 1994.

..S. Wagner, p. 47 in Whisker Technology (A.P. Levitt, ed.) Wiley, New York, 1970.

..I. Givargizov, Current Topics in Materials Science 1, 79 (1978).

P. Hirth and J. M. Pound, Progr. Mater. Sci. 11, 107 (1963). 


\section{Figure Captions}

Fig. 1 (Top) SEM image of Si microcolumns formed after 1000 laser shots in air at $E_{d}=3 \mathrm{~J} / \mathrm{cm}^{2}$ (scale bar $=100 \mu \mathrm{m})$. (Bottom) Droplets formed at the tips of silicon microcolumns (scale bar $=10 \mu \mathrm{m})$.

Fig. 2 (a) Surface-height profile across the narrow direction of the laser-irradiated area. (b) SEM image showing pronounced redeposition of ablated material just outside the laserirradiated region (to the left of the dashed line) as seen also in the surface-height profile.

Fig. 3 Steeply sloping Si cones connected by walls that were formed in $1 \mathrm{~atm}$. of SF6. (Top) 2000 laser pulses at $E_{d}=2.9 \mathrm{~J} / \mathrm{cm}^{2}$. (Bottom) 2040 laser pulses at $E_{d}=1.5 \mathrm{~J} / \mathrm{cm}^{*}$. In each case the higher magnification view on the right shows the features decorating the cones and adjoining walls.

Fig. 4 Surface fracturing of silicon laser-irradiated in air. (Top) (001)Si after 250 pulses at 3.3 $\mathrm{J} / \mathrm{cm}^{*}$. (Bottom) (11 1)Si after 200 pulses at $3.2 \mathrm{~J} / \mathrm{cm}^{2}$.

Fig. 5 Microcolumns formed in air on (001)Si by $400 \mathrm{KrF}$ laser pulses at $\mathrm{E}_{\mathrm{d}}=2.7 \mathrm{~J} / \mathrm{cm}^{2}$. The scale bars represent $10 \mu \mathrm{m}$.

Fig. 6 Comparison of surface evolution for (001)Si laser-irradiated at $3 \mathrm{~J} / \mathrm{cm}^{2}$ in SF, (left side) and in air (right side). From top to bottom the number of laser pulses in SF, (in air) are: $250(250), 500(500)$ and $7.50(600)$.

Fig. 7. Disordered intersecting ring structure formed after 500 laser pulses at $3 \mathrm{~J} / \mathrm{cm}^{2}$ in $\mathrm{SF}$, showing the "dome" that emerges from the intersection of several ring segments.

Fig. 8. (a) Globular features produced by pulsed-laser irradiation of (001)Si in pure oxygen. Surface-height profiles show (b) initial swelling after only 25 laser shots and (c) etching of the central region after 125 laser shots.

Fig. 9. (a) Initial formation (500 shots) and (b) final morphology (10,000 shots) of "lateral cones" or "thorns" grown on the side walls of microcones by pulsed-laser irradiation of $\mathrm{Si}$ in $\mathrm{SF}$,

Fig. 10. Surface ripple pattern produced by irradiating $\mathrm{Si}(\mathrm{OO} 1)$ with $2000 \mathrm{KrF}$ laser shots at 3 $\mathrm{J} / \mathrm{cm}^{2}$ in 0.76 torr of oxygen. 

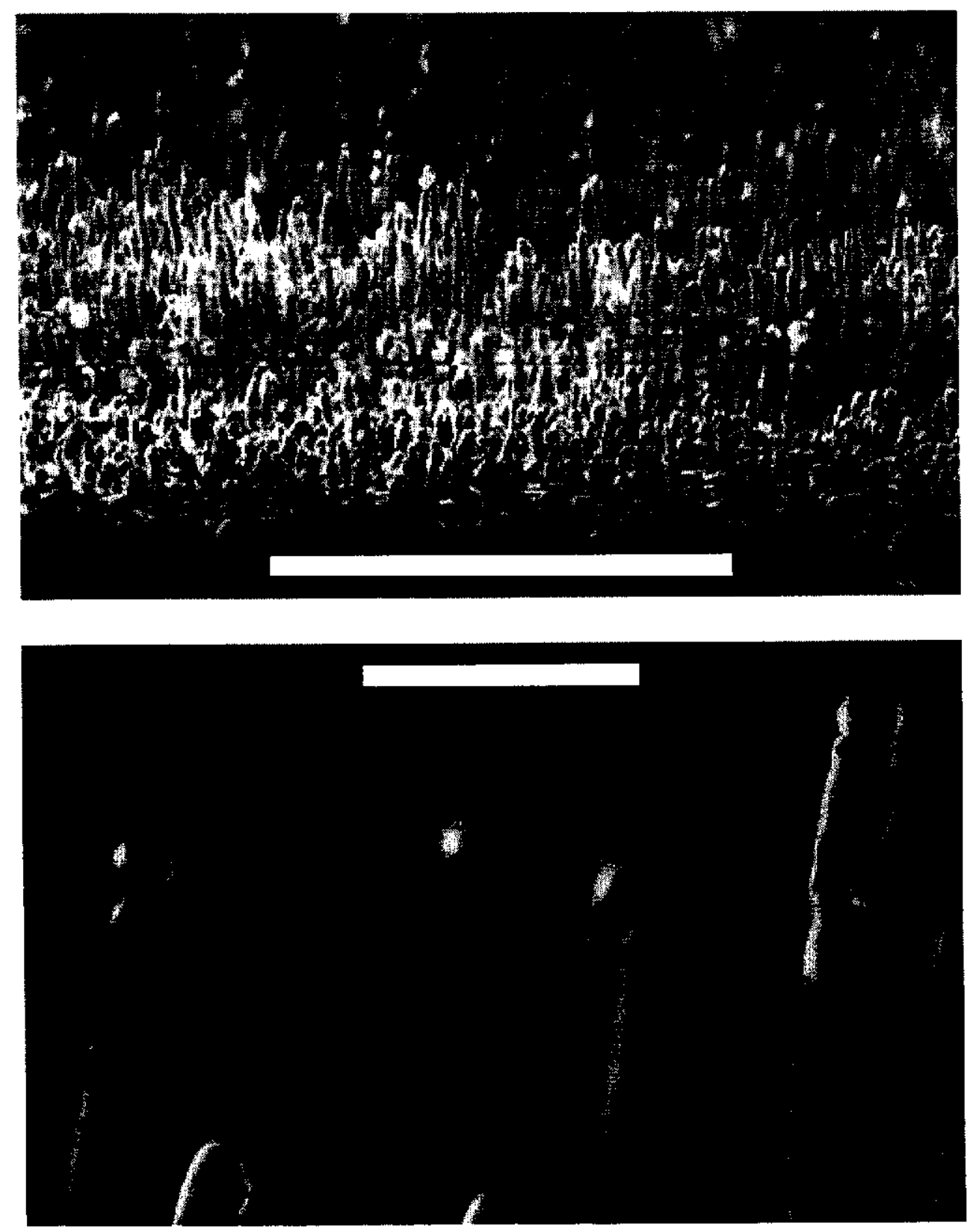

Figure 1 

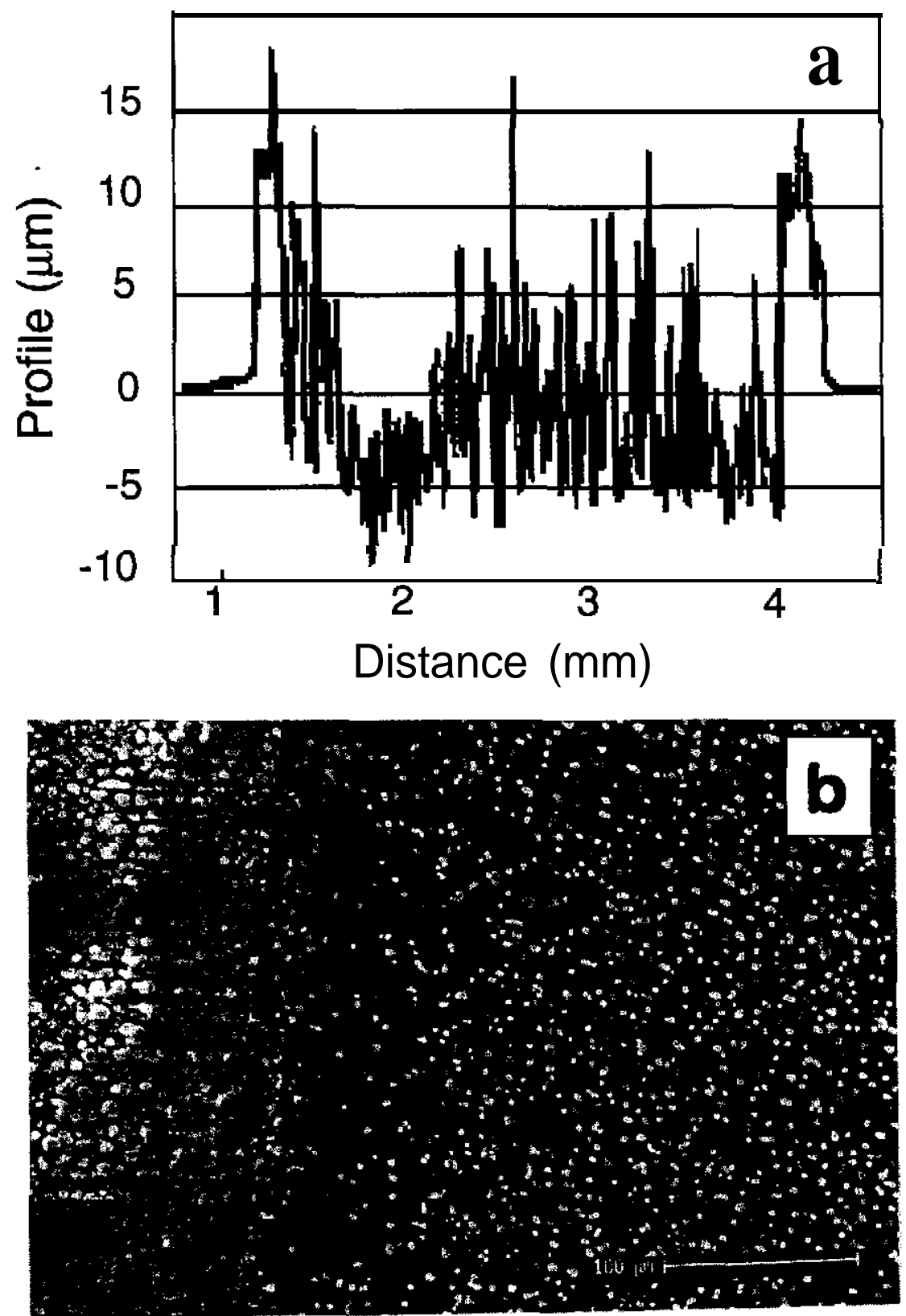

Figure 2 

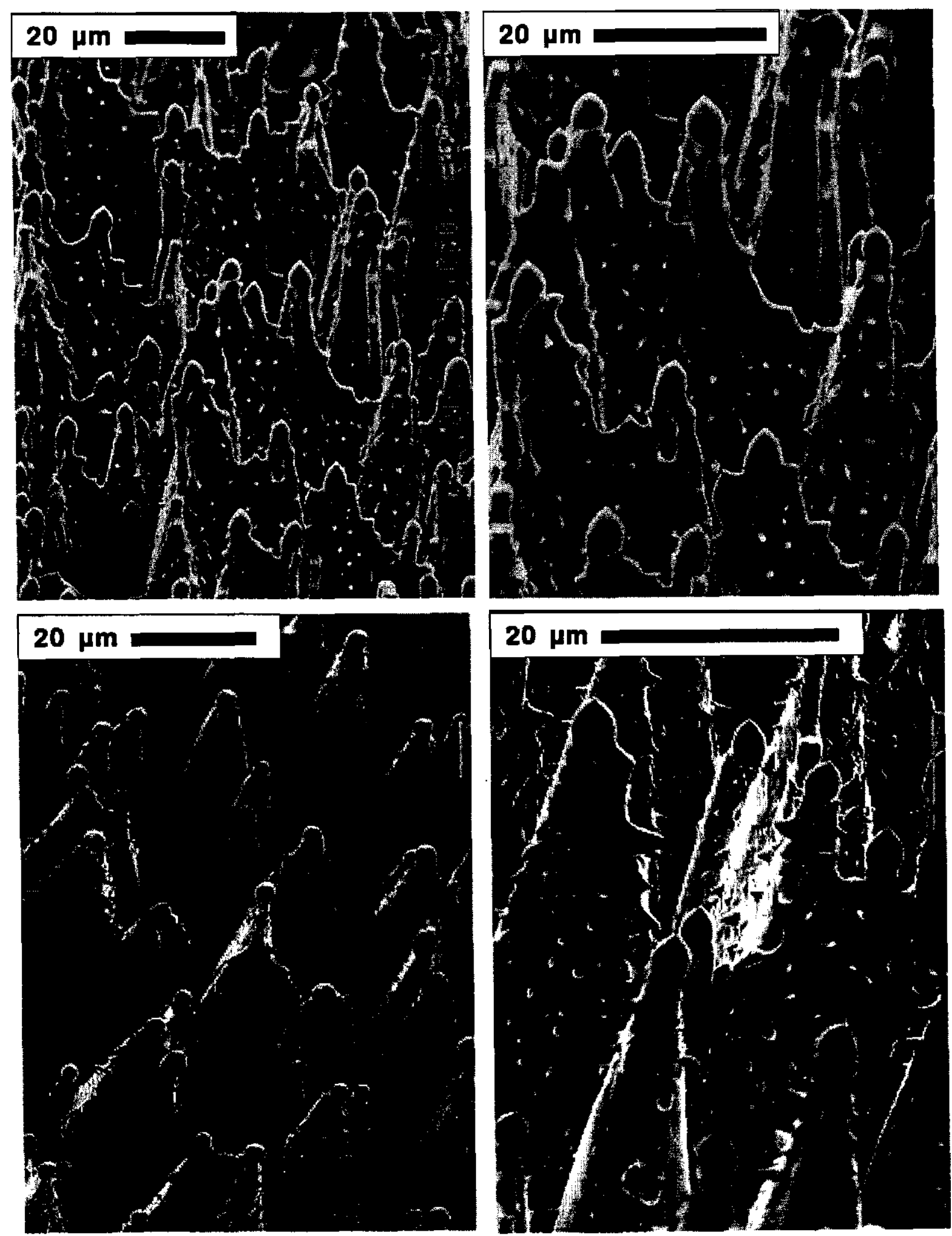

Figure 3 

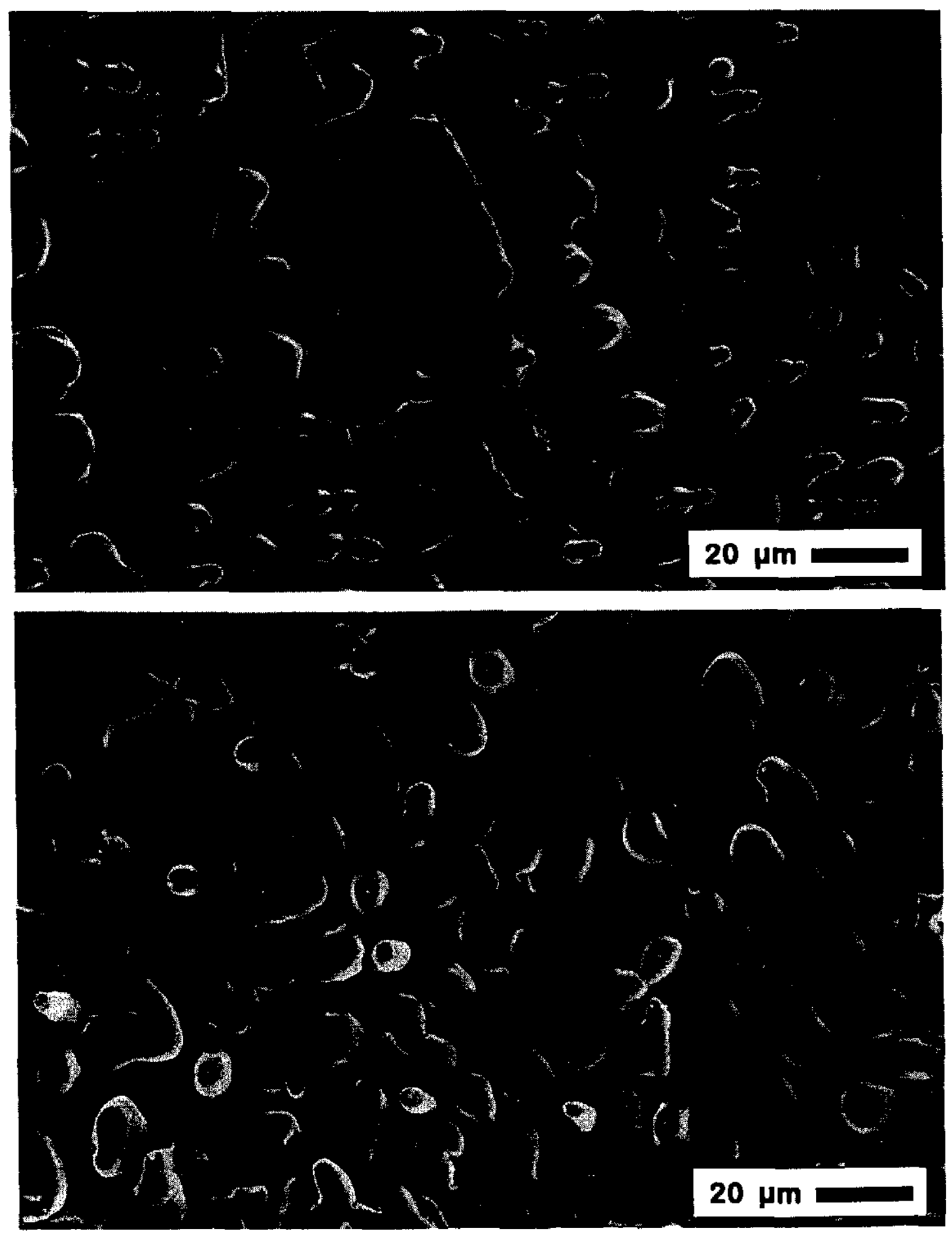

Figure 4 


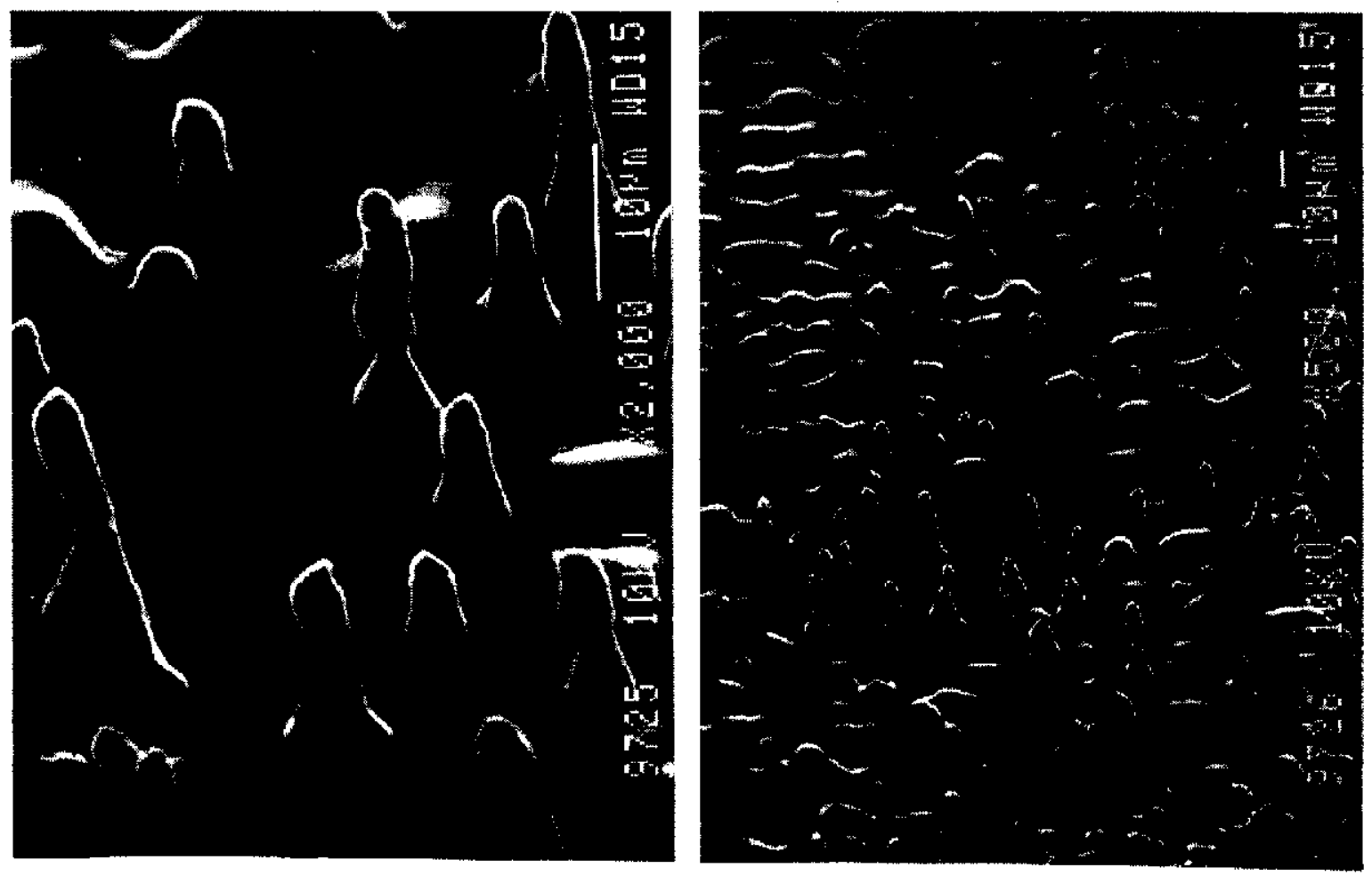

Figure 5 


\section{$\frac{5}{8}$}
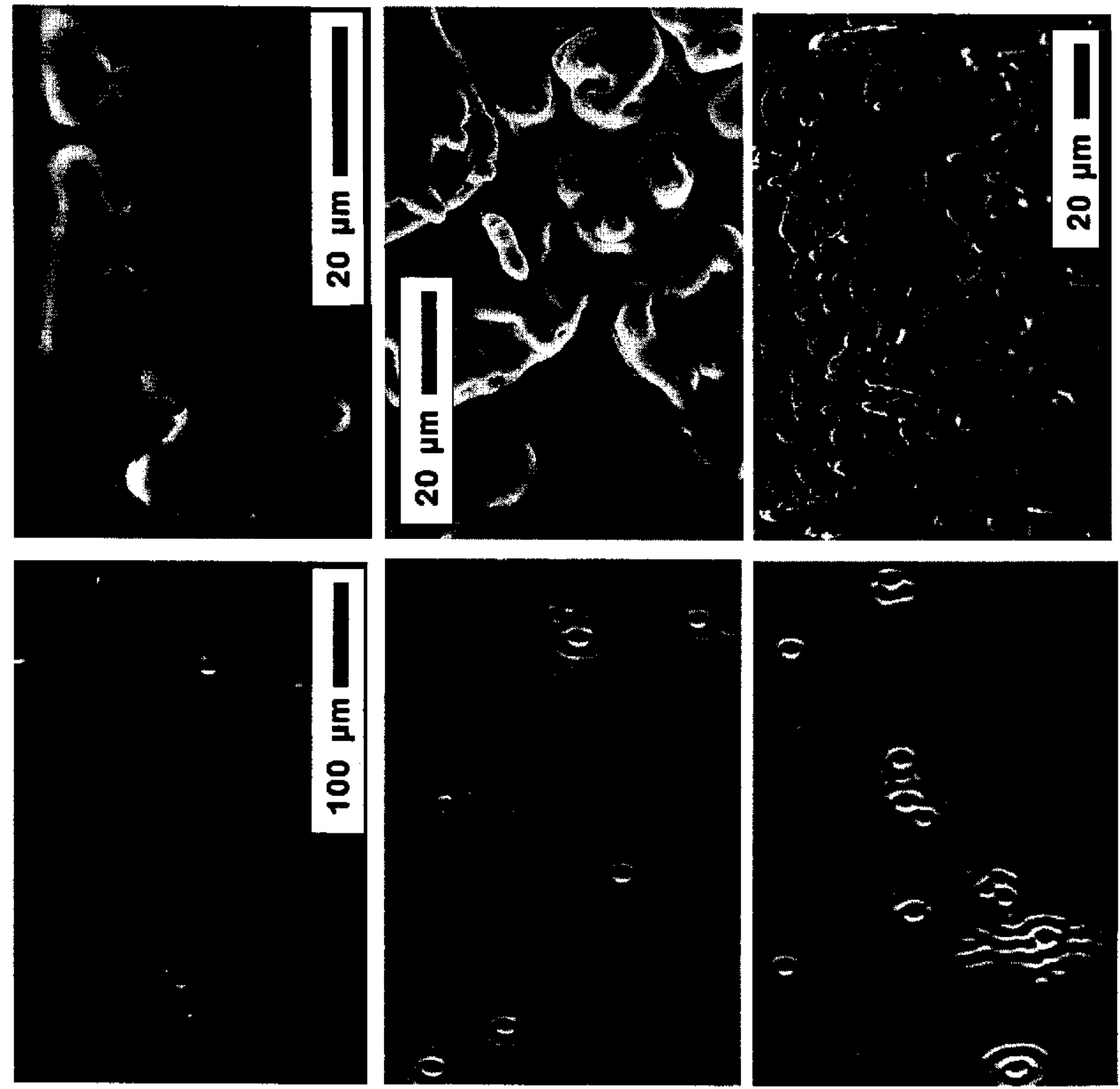

0
6
5
11

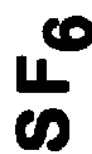



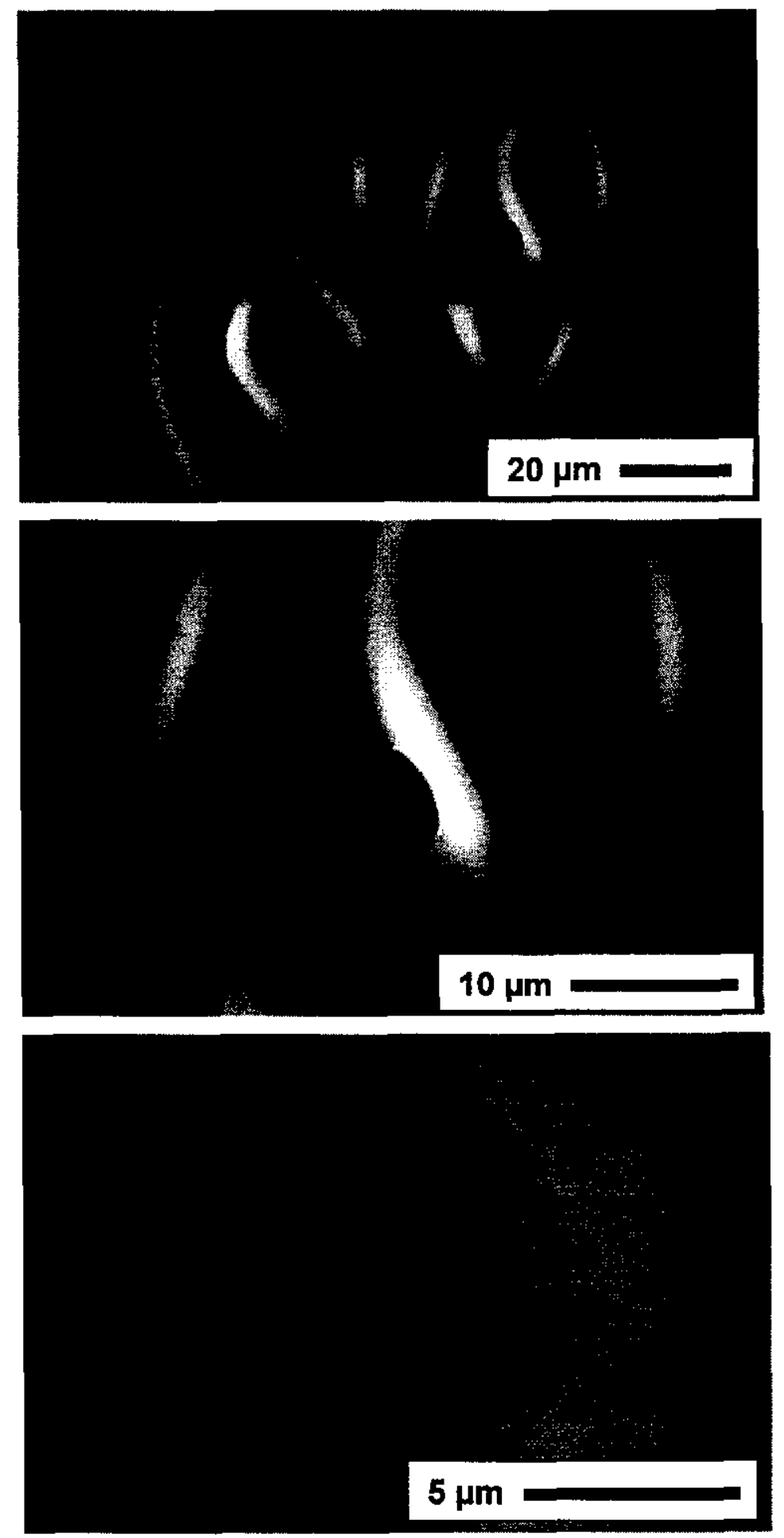

Figure 7 

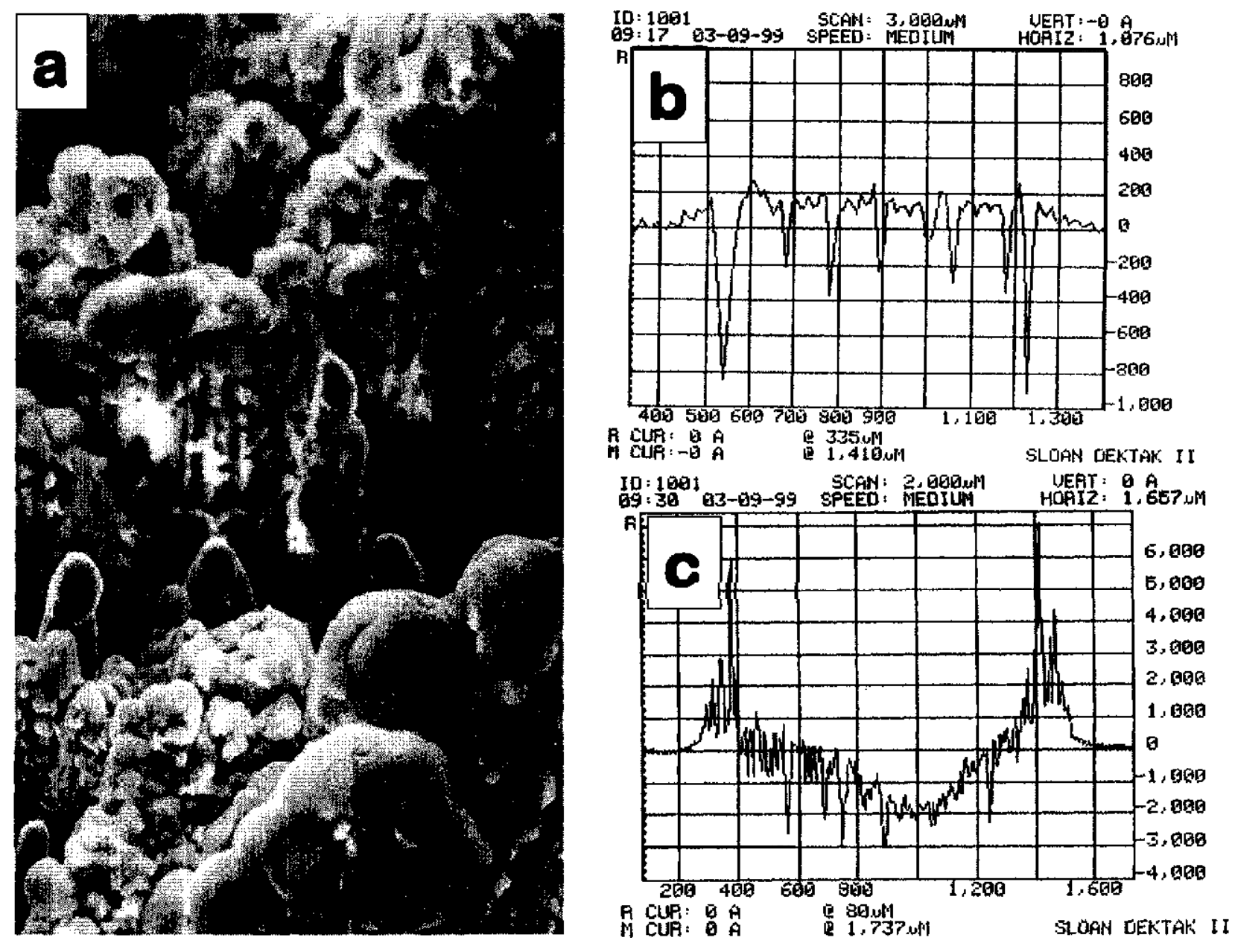

Figure 8 


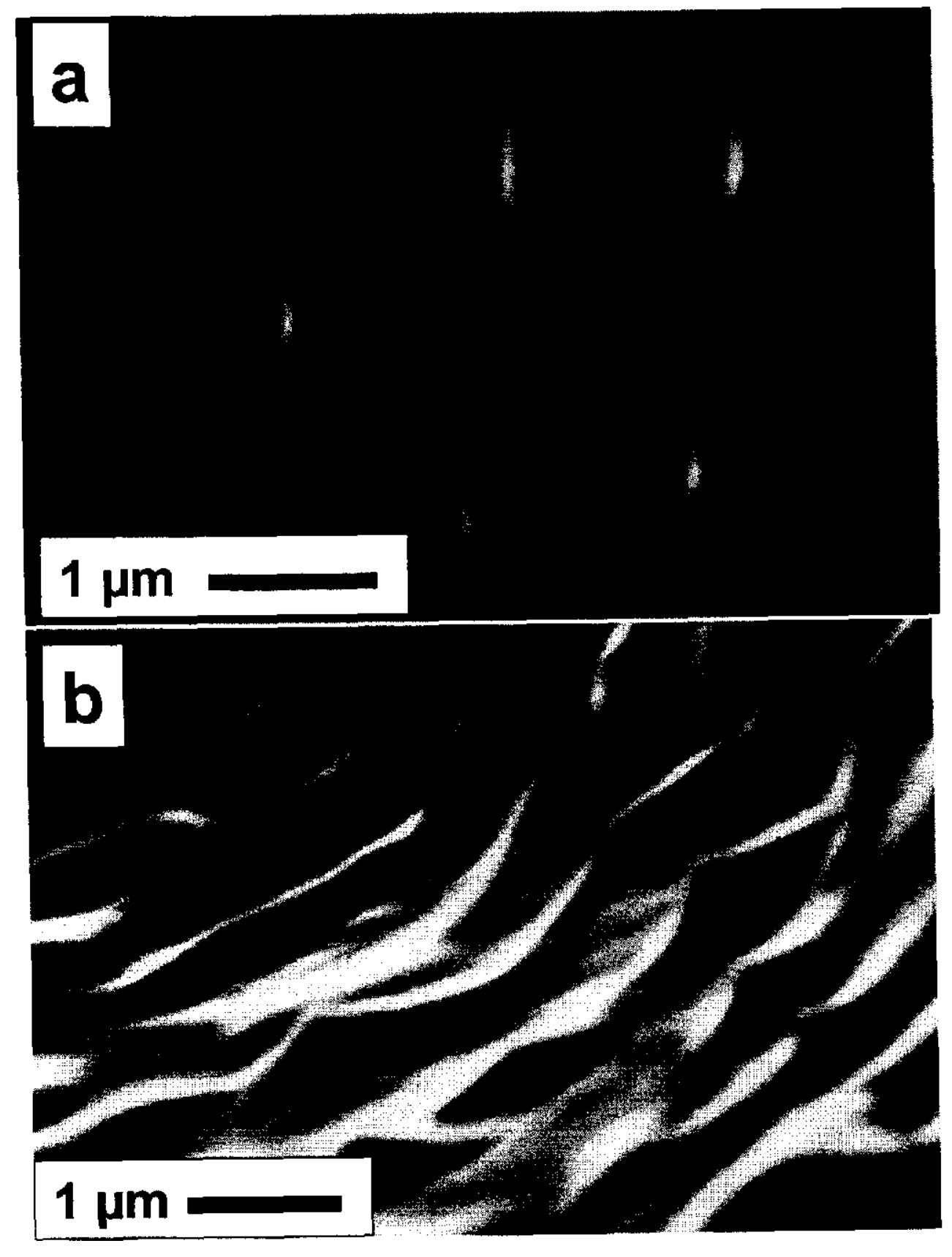

Figure 9 


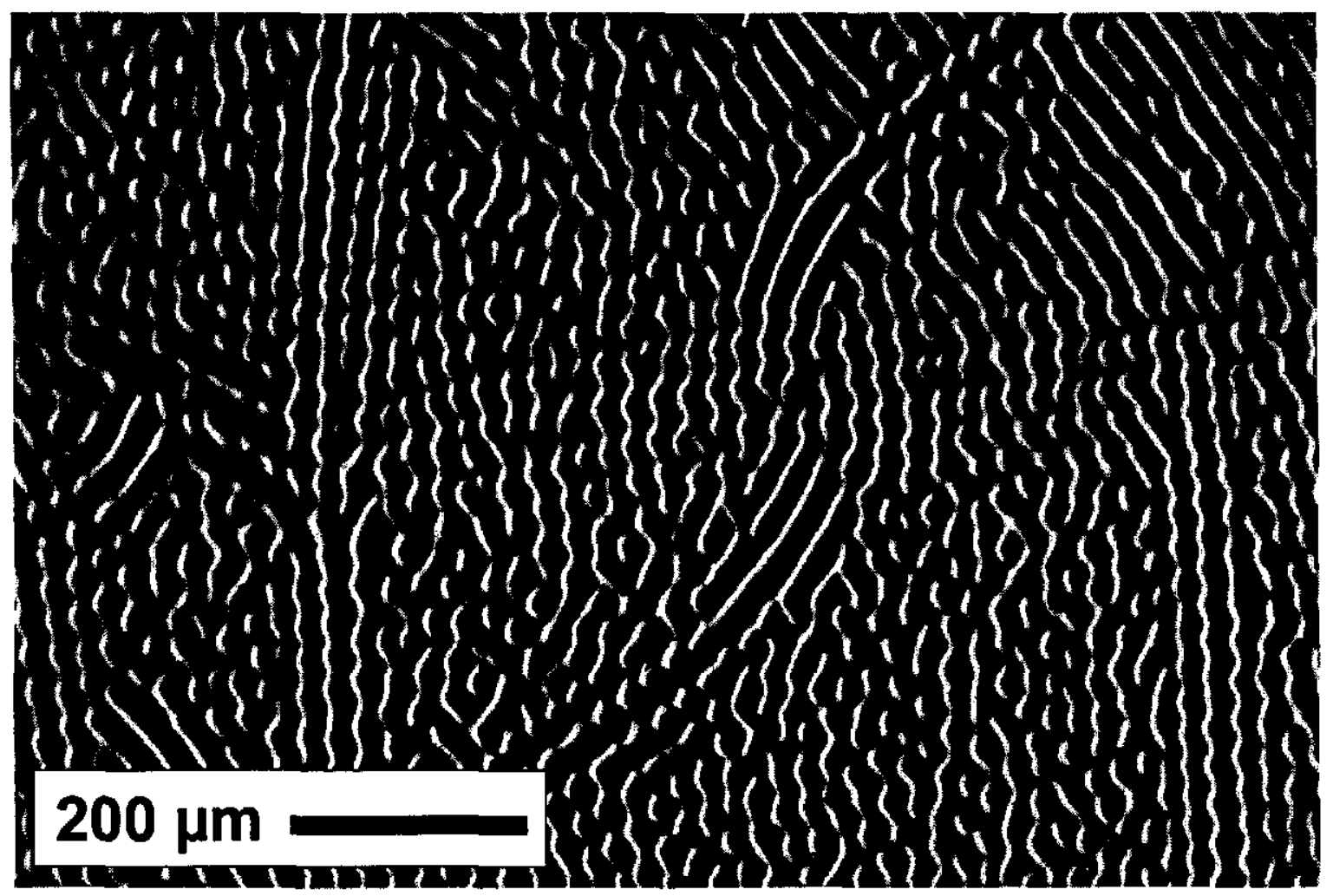

Figure 10 